PROCEEDINGS OF THE

AMERICAN MATHEMATICAL SOCIETY

Volume 127, Number 2, February 1999, Pages 499-507

S 0002-9939(99)04597-9

\title{
INTERPOLATION IN INFLATED HILBERT SPACES
}

\author{
R. L. MOORE AND T. T. TRENT
}

(Communicated by Palle E. T. Jorgensen)

\begin{abstract}
The interpolation problem for a reflexive algebra $\operatorname{Alg} \mathcal{L}$ is this: Given two operators $X$ and $Y$, under what conditions can we be sure that there will exist an operator $A$ in $A \lg \mathcal{L}$ such that $A X=Y$ ? There are simple necessary conditions that have been investigated in several earlier papers. Here we present an example to show that the conditions are not, in general, sufficient. We also suggest a strengthened set of conditions which are necessary and are "almost" sufficient, in the sense that they will ensure that $Y$ lies in the weak-operator closure of the set $\{A X: A \in A \lg \mathcal{L}\}$.
\end{abstract}

Given a Hilbert space $\mathcal{H}$, a subalgebra $\mathcal{A}$ of operators acting on $\mathcal{H}$, and operators $X$ and $Y$ in $B(\mathcal{H})$ (not necessarily in $\mathcal{A}$ ), how can we know whether there is an operator $A \in \mathcal{A}$ such that $A X=Y$ ? There is an easy necessary condition, a special case of which was investigated by Arveson [1], Lance [5] and later by Hopenwasser [3]. Those authors were interested in the case that $\mathcal{A}$ is a reflexive algebra, and that $X$ and $Y$ are rank-one. Suppose that there exists an $A \in \mathcal{A}$ such that $A X=Y$, and let $E$ be any projection in $L a t \mathcal{A}$, that is, the lattice of projections invariant for the algebra $\mathcal{A}$. Then, for any $B \in \mathcal{A}, E^{\perp} B E^{\perp}=E^{\perp} B$, and we have

$$
\begin{aligned}
E^{\perp} A X & =E^{\perp} Y \quad \text { and consequently, for each } f \in \mathcal{H}, \\
E^{\perp} A E^{\perp} X f & =E^{\perp} Y f . \quad \text { It follows that } \\
\left\|E^{\perp} Y f\right\| & \leq\|A\|\left\|E^{\perp} X f\right\|, \quad \text { or } \\
\sup _{\substack{E \in L_{a t} \mathcal{A} \\
f \in \mathcal{H}}} \frac{\left\|E^{\perp} Y f\right\|}{\left\|E^{\perp} X f\right\|} & \leq\|A\| .
\end{aligned}
$$

For any two operators $X$ and $Y$, let $\rho(X, Y)$ represent the quantity on the left-hand side above:

$$
\rho(X, Y)=\sup \left\{\frac{\left\|E^{\perp} Y f\right\|}{\left\|E^{\perp} X f\right\|}: E \in \text { Lat } \mathcal{A} \text { and } f \in \mathcal{H}\right\} .
$$

(We use the convention that $0 / 0=0$ for the purposes of computing the supremum.) Then, a necessary condition for the existence of an operator $A \in \mathcal{A}$ such that $A X=Y$ is that $\rho(X, Y)<\infty$. Furthermore, if such an $A$ exists, it is clear that $\|A\| \geq \rho(X, Y)$.

A subspace lattice $\mathcal{L}$ is a collection of projections acting on $\mathcal{H}$, closed in the strong operator topology, and also closed under the usual meet and join operations. The

Received by the editors June 13, 1996 and, in revised form, May 27, 1997.

1991 Mathematics Subject Classification. Primary 47D25.

(C)1999 American Mathematical Society 
associated reflexive algebra, $A \lg \mathcal{L}$, is the collection of all operators leaving invariant each of the projections in $\mathcal{L}$. If the projections in $\mathcal{L}$ commute pairwise, then $\mathcal{L}$ is a commutative subspace lattice (CSL), and if $\mathcal{L}$ is totally ordered by inclusion (and hence commutative) then $\mathcal{L}$ is a nest. In these cases the algebra $A \lg \mathcal{L}$ is referred to as a CSL algebra or a nest algebra.

Suppose now that the operators $X$ and $Y$ are rank-one. Lance's result is that, if $\mathcal{L}$ is a nest, then the condition $\rho(X, Y)<\infty$ is sufficient to ensure the existence of an operator $A \in A \lg \mathcal{L}$ such that $A X=Y$. Hopenwasser's result is that the same is true, even if the lattice $\mathcal{L}$ is simply assumed to be a CSL. Moreover, in each of these results, it was shown that the operator $A$ can be chosen to have norm equal to $\rho(X, Y)$. Motivated by the work of these authors, Elias Katsoulis and we have shown that, if $\mathcal{L}$ is a nest, the same result is true even if there is no restriction on the rank of the operators $X$ and $Y$.

The purpose of this paper is to proceed with the investigation of the condition $\rho(X, Y)<\infty$. In particular, what if $\mathcal{L}$ is not assumed to be a nest, and, in addition, no assumption is made about the rank of the operators $X$ and $Y$ ? Our main result will show that the condition $\rho(X, Y)<\infty$ is no longer sufficient to guarantee the existence of the interpolating operator $A$. The main example will operate on an infinite-dimensional Hilbert space, but we first present a finite-dimensional example that will show that the norm of $A$ might have to be larger than $\rho(X, Y)$.

Example. Let $\mathcal{H}$ be the three-dimensional Hilbert space $\mathbb{C}^{3}$, with orthonormal basis $\left\{e_{1}, e_{2}, e_{3}\right\}$. Let $\mathcal{L}_{0}$ be the lattice $\left\{0, P_{1}, P_{2}, P_{3}, I\right\}$, where $P_{1}$ and $P_{2}$ are the projections whose ranges are the one-dimensional spaces spanned by $e_{1}$ and $e_{2}$ respectively, and $P_{3}=P_{1} \vee P_{2}$. The operators in $A \lg \mathcal{L}$ all have the form

$$
A=\left[\begin{array}{lll}
\alpha & 0 & \beta \\
0 & \gamma & \delta \\
0 & 0 & \epsilon
\end{array}\right]
$$

Let

$$
X=\left[\begin{array}{ccc}
0 & \sqrt{2} & 1 \\
0 & 1 & 0 \\
0 & 0 & 1
\end{array}\right] \quad \text { and } \quad Y=\left[\begin{array}{lll}
0 & 1 & 0 \\
0 & 0 & 1 \\
0 & 0 & 0
\end{array}\right]
$$

It is straightforward to compute that, for each $E \in \mathcal{L}_{0}, X^{*} E^{\perp} X \geq Y^{*} E^{\perp} Y$; this is the same as saying that $\left\|E^{\perp} X f\right\| \geq\left\|E^{\perp} Y f\right\|$ for every $E \in \mathcal{L}_{0}$ and every $f \in \mathcal{H}$. Furthermore, $\left\|P_{3}^{\perp} X e_{3}\right\|=\left\|P_{3}^{\perp} Y e_{3}\right\|$. Therefore, $\rho(X, Y)=1$. On the other hand, the only matrix in $A \lg \mathcal{L}_{0}$ that solves the equation $A X=Y$ is

$$
A=\left[\begin{array}{ccc}
1 / \sqrt{2} & 0 & -1 / \sqrt{2} \\
0 & 0 & 1 \\
0 & 0 & 0
\end{array}\right],
$$

which, quite obviously, has norm greater than 1 .

We base our main construction on this example. Our intention is to find operators $X$ and $Y$, and a lattice $\mathcal{L}$, acting on an infinite-dimensional Hilbert space, so that $\rho(X, Y)=1$ but so that there is no bounded operator $A \in A \lg \mathcal{L}$ such that $A X=Y$. This will show that, for some lattices, there is not even a constant $K$ such that the condition $\rho(X, Y)<\infty$ implies the existence of an operator $A$ so that $A X=Y$ and $\|A\| \leq K \cdot \rho(X, Y)$. 
Lemma 1. For any bounded operator $A$ acting on $\mathcal{H}$, let

$$
B=\left[\begin{array}{rrr}
A & 0 & -A \\
0 & 0 & I \\
0 & 0 & 0
\end{array}\right] \text {. }
$$

Then $\|B\|^{2} \geq \frac{1}{2}\left[1+2\|A\|^{2}+\sqrt{1+4\|A\|^{4}}\right]$.

Proof. Choose any vector $f \in \mathcal{H}$ so that $\|f\|=1$ and $\|A f\|$ is near to $\|A\|$. For any complex number $\lambda$, the vector $[\lambda f, 0, f]$ has norm $\sqrt{1+|\lambda|^{2}}$. If we apply $B$ to this vector, we get $[(\lambda-1) A f, f, 0]$, whose norm is $\sqrt{1+|\lambda-1|^{2}\|A\|^{2}}$. Thus,

$$
\|B\|^{2} \geq \sup _{\lambda} \frac{1+|\lambda-1|^{2}\|A\|^{2}}{1+|\lambda|^{2}} .
$$

The result follows in a straightforward fashion. One way to see this is to observe that the last expression is also equal to the norm of the $2 \times 2$ matrix

$$
\left[\begin{array}{cc}
\|A\| & -\|A\| \\
0 & 1
\end{array}\right]
$$

Actually, the norm of $B$ is equal to the expression above, but we'll only need the inequality.

Corollary. For any operator $A$ in $B(\mathcal{H})$, let $\tilde{A}$ be the matrix

$$
\tilde{A}=\left[\begin{array}{ccc}
(1 / \sqrt{2}) A & 0 & (-1 / \sqrt{2}) A \\
0 & 0 & I \\
0 & 0 & 0
\end{array}\right] .
$$

Then $\|\tilde{A}\|^{2} \geq\|A\|^{2}+\frac{1}{2}$.

Proof. This fact follows from the result above and the fact that $\sqrt{1+x^{2}} \geq x$ for all positive $x$.

Lemma 2. For any $A \in B(\mathcal{K})$, let $\tilde{A}$ be the operator acting on $\mathcal{K} \otimes \mathbb{C}^{3}$ given as above. Let $A_{1}=A, A_{2}=\tilde{A}_{1}, A_{3}=\tilde{A}_{2}$, and so on. This provides a sequence of operators $\left\{A_{n}\right\}_{n=1}^{\infty}$ acting on successively larger Hilbert spaces. Then $\left\|A_{n}\right\| \rightarrow \infty$, as long as $A \neq 0$.

Proof. It follows from the corollary that $\left\|A_{2}\right\|^{2} \geq\|A\|^{2}+\frac{1}{2}, \quad\left\|A_{3}\right\|^{2} \geq\left\|A_{2}\right\|^{2}+\frac{1}{2} \geq$ $\|A\|^{2}+2 \cdot \frac{1}{2}, \ldots$

We are going to build a sequence of lattices based on the one in the simple counterexample given earlier. Each lattice will be the tensor product of the previous one with the lattice of the counterexample. Let $\mathbb{C}^{3}$ be given an orthonormal basis $\left\{e_{1}, e_{2}, e_{3}\right\}$. Define projections $P_{i}$ on $C^{3}$ as follows:

$$
\begin{aligned}
P_{1} & =\left[e_{1}\right], \\
P_{2} & =\left[e_{2}\right], \\
P_{3} & =\left[e_{1}, e_{2}\right],
\end{aligned}
$$

where the square bracket means, for instance, that $P_{3}$ is the orthogonal projection onto the span of the vectors $e_{1}$ and $e_{2}$. 
Let $\mathcal{L}_{0}$ be the lattice $\left\{0, P_{1}, P_{2}, P_{3}, I\right\}$. Now, for any commutative subspace lattice $\mathcal{L}$ of projections acting on a Hilbert space $\mathcal{K}$, we can form the lattice $\mathcal{L} \otimes \mathcal{L}_{0}$, which acts on the space $\mathcal{K} \otimes \mathbb{C}^{3}$; this lattice is the smallest CSL that contains all the elementary tensors. Any operator on $\mathcal{K} \otimes \mathbb{C}^{3}$ can be represented as a $3 \times 3$ matrix whose entries are operators on $\mathcal{K}$, and, with this understanding, we have the following theorem.

Lemma 3. Let $\mathcal{L}$ be any $C S L$ acting on a Hilbert space $\mathcal{K}$. Then any projection in $\mathcal{L} \otimes \mathcal{L}_{0}$ has the form

$$
\left[\begin{array}{ccc}
E & 0 & 0 \\
0 & F & 0 \\
0 & 0 & E F G
\end{array}\right]
$$

where $E, F, G \in \mathcal{L}$.

Proof. Note that the collection of all matrices formed as indicated is indeed a complete lattice of subspaces. Furthermore, the tensor product $\mathcal{L} \otimes \mathcal{L}_{0}$ is generated by spans of projections of the form $E_{1} \otimes P_{1}, E_{2} \otimes P_{2}, E_{3} \otimes P_{3}$, and $E_{4} \otimes I$. Matricially, these look like

$$
\begin{array}{ll}
{\left[\begin{array}{ccc}
E_{1} & 0 & 0 \\
0 & 0 & 0 \\
0 & 0 & 0
\end{array}\right],} & {\left[\begin{array}{ccc}
0 & 0 & 0 \\
0 & E_{2} & 0 \\
0 & 0 & 0
\end{array}\right],} \\
{\left[\begin{array}{ccc}
E_{3} & 0 & 0 \\
0 & E_{3} & 0 \\
0 & 0 & 0
\end{array}\right],} & {\left[\begin{array}{ccc}
E_{4} & 0 & 0 \\
0 & E_{4} & 0 \\
0 & 0 & E_{4}
\end{array}\right] .}
\end{array}
$$

Any span of such things has the form specified in the lemma. Thus, it remains only to show that any matrix with the specified form is a span of elementary tensor products in $\mathcal{L} \otimes \mathcal{L}_{0}$. But this fact is clear, since we can take $E_{3}=0, E_{1}=E$, $E_{2}=F$, and $E_{4}=E F G$.

Our plan is to build successively larger Hilbert spaces by forming tensor products with $\mathbb{C}^{3}$. The lattice acting on each space will be a tensor product of the previous lattice with $\mathcal{L}_{0}$. Suppose that $\mathcal{L}$ is a commutative subspace lattice acting on a space $\mathcal{K}$; we will use the notation $\hat{\mathcal{K}}$ to represent the tensor product $\mathcal{K} \otimes \mathbb{C}^{3}$, and, by $\hat{\mathcal{L}}$, we mean the tensor product $\mathcal{L} \otimes \mathcal{L}_{0}$. If $X$ and $Y$ are operators on the space $\mathcal{K}$, we define operators $\hat{X}$ and $\hat{Y}$ acting on $\hat{\mathcal{K}}$ by

$$
\hat{X}=\left[\begin{array}{ccc}
0 & \sqrt{2} X & I \\
0 & X & 0 \\
0 & 0 & I
\end{array}\right] \quad \text { and } \quad \hat{Y}=\left[\begin{array}{ccc}
0 & Y & 0 \\
0 & 0 & I \\
0 & 0 & 0
\end{array}\right]
$$

The notation is inconsistent, but we prefer it to a multiplicity of different mathematical accents; hats simply act differently on things named $X$ and $Y$ and $E$, and they will never act on anything else. The theorem that follows says simply that, if operators $X$ and $Y$ satisfy the condition $\rho(X, Y)<\infty$ for a lattice $\mathcal{L}$, then the new operators $\hat{X}$ and $\hat{Y}$ satisfy the conditions for the lattice $\hat{\mathcal{L}}$.

Theorem 1. Suppose that, for all $E \in \mathcal{L}$ and for all vectors $f \in \mathcal{K}$, we have

$$
\left\|E^{\perp} Y f\right\| \leq\left\|E^{\perp} X f\right\| .
$$


Then, for all $\hat{E} \in \hat{\mathcal{L}}$, and for all $\hat{f} \in \hat{\mathcal{K}}$, it is also true that

$$
\left\|\hat{E}^{\perp} \hat{Y} \hat{f}\right\| \leq\left\|\hat{E}^{\perp} \hat{X} \hat{f}\right\| .
$$

Proof. The condition $\rho(X, Y), \infty$ is equivalent to the conditions $X^{*} E^{\perp} X \geq Y^{*} E \perp Y$ for all $E \in \mathcal{L}$. Thus, we need to show that $\hat{X}^{*} \hat{E}^{\perp} \hat{X} \geq \hat{Y}^{*} \hat{E}^{\perp} \hat{Y}$ for all $\hat{E} \in \hat{\mathcal{L}}$. According to the last lemma, the matricial version is

$$
\begin{aligned}
& {\left[\begin{array}{ccc}
0 & 0 & 0 \\
\sqrt{2} X^{*} & X^{*} & 0 \\
I & 0 & I
\end{array}\right]\left[\begin{array}{ccc}
E^{\perp} & 0 & 0 \\
0 & F^{\perp} & 0 \\
0 & 0 & (E F G)^{\perp}
\end{array}\right]\left[\begin{array}{ccc}
0 & \sqrt{2} X & I \\
0 & X & 0 \\
0 & 0 & I
\end{array}\right]} \\
& \geq\left[\begin{array}{ccc}
0 & 0 & 0 \\
Y^{*} & 0 & 0 \\
0 & I & 0
\end{array}\right]\left[\begin{array}{ccc}
E^{\perp} & 0 & 0 \\
0 & F^{\perp} & 0 \\
0 & 0 & (E F G)^{\perp}
\end{array}\right]\left[\begin{array}{ccc}
0 & Y & 0 \\
0 & 0 & I \\
0 & 0 & 0
\end{array}\right] .
\end{aligned}
$$

Because of the shape of the matrices, the last inequality is equivalent to a $2 \times 2$ condition:

$$
\left[\begin{array}{cc}
2 X^{*} E^{\perp} X-Y^{*} E^{\perp} Y+X^{*} F^{\perp} X & \sqrt{2} X^{*} E^{\perp} \\
\sqrt{2} E^{\perp} X & E^{\perp}+(E F G)^{\perp}-F^{\perp}
\end{array}\right] \geq 0 .
$$

We refer to the matrix above as $\phi(E, F, G)$ and note that, for all projections $E, F$, and $G$,

$$
\phi(E, F, G)=\phi(E, F, I)+\left[\begin{array}{cc}
0 & 0 \\
0 & (E F G)^{\perp}-(E F)^{\perp}
\end{array}\right] .
$$

Since the last matrix is always positive, it will suffice to show that $\phi(E, F, I) \geq 0$ for all $E, F \in \mathcal{L}$. Because $\rho(X, Y)<\infty,, X^{*} E^{\perp} X-Y^{*} E^{\perp} Y \geq 0$, so we can write

$$
\begin{aligned}
\phi(E, F, I)= & {\left[\begin{array}{cc}
X^{*} E^{\perp} X+X^{*} F^{\perp} X & \sqrt{2} X^{*} E^{\perp} \\
\sqrt{2} E^{\perp} X & E^{\perp}-F^{\perp}+(E F)^{\perp}
\end{array}\right] } \\
& +\left[\begin{array}{cc}
X^{*} E^{\perp} X-Y^{*} E^{\perp} Y & 0 \\
0 & 0
\end{array}\right] .
\end{aligned}
$$

It suffices to show that the first matrix above is positive for all $E$. That matrix, however, is the same as

$$
\left[\begin{array}{cc}
X^{*} & 0 \\
0 & I
\end{array}\right]\left[\begin{array}{cc}
E^{\perp}+F^{\perp} & \sqrt{2} E^{\perp} \\
\sqrt{2} E^{\perp} & E^{\perp}-F^{\perp}+(E F)^{\perp}
\end{array}\right]\left[\begin{array}{cc}
X & 0 \\
0 & I
\end{array}\right] .
$$

If we can see why the center matrix in the display above is positive for all $E$ and $F$, we will be done. Since the projections commute, they can be considered to lie in some abelian $\mathrm{C}^{*}$-algebra $C(\mathrm{X})$. Therefore, we can do the computation pointwise (in $\mathrm{X})$. Let $x \in \mathrm{X}$ and set $e=E^{\perp}(x), f=F^{\perp}(x)$; then $\left(E^{\perp}-F^{\perp}+(E F)^{\perp}\right)(x)=$ $2 e-f e$. The real matrix

$$
\left[\begin{array}{cc}
e+f & \sqrt{2} e \\
\sqrt{2} e & 2 e-f e
\end{array}\right]
$$

is easily seen to be positive, and the proof is complete.

We are now ready for our counterexample. To be precise, there exist a commutative subspace lattice $\mathcal{L}$ and operators $X$ and $Y$ such that

$$
Y^{*} E^{\perp} Y \leq X^{*} E^{\perp} X \quad \text { for each } E \in \mathcal{L}
$$

and yet, there is no bounded operator $A \in A \lg \mathcal{L}$ for which $A X=Y$. 
Let

$$
X_{1}=\left[\begin{array}{ccc}
0 & \sqrt{2} & I \\
0 & I & 0 \\
0 & 0 & I
\end{array}\right] \quad \text { and } \quad Y_{1}=\left[\begin{array}{ccc}
0 & I & 0 \\
0 & 0 & I \\
0 & 0 & 0
\end{array}\right]
$$

and, for any $X$ and $Y$, we let, as before,

$$
\hat{X}=\left[\begin{array}{ccc}
0 & \sqrt{2} X & I \\
0 & X & 0 \\
0 & 0 & I
\end{array}\right] \quad \text { and } \quad \hat{Y}=\left[\begin{array}{ccc}
0 & Y & 0 \\
0 & 0 & I \\
0 & 0 & 0
\end{array}\right]
$$

For any lattice $\mathcal{L}$, let $\hat{\mathcal{L}}$ be $\mathcal{L} \otimes \mathcal{L}_{0}$, where $\mathcal{L}_{0}$ is as usual. Form sequences as follows:

$$
\begin{array}{lll}
X_{2}=\hat{X}_{1} & Y_{2}=\hat{Y}_{1} & \mathcal{L}_{2}=\hat{\mathcal{L}}_{1} \\
X_{3}=\hat{X}_{2} & Y_{3}=\hat{Y}_{2} & \mathcal{L}_{3}=\hat{\mathcal{L}}_{2} \\
X_{4}=\hat{X}_{3} & Y_{4}=\hat{Y}_{3} & \mathcal{L}_{4}=\hat{\mathcal{L}}_{3}
\end{array}
$$

Then, for each $j$, the operators $X_{j}$ and $Y_{j}$ and the lattice $\mathcal{L}_{j}$ all act on the finitedimensional Hilbert space $\mathbb{C}^{3^{j}}$. Suppose that, for some value of $j$, there exists $A_{j} \in \operatorname{Alg}(\mathcal{L})$ for which

$$
A_{j+1} X_{j+1}=Y_{j+1} .
$$

The matricial representation of this equation is

$$
\left[\begin{array}{ccc}
A & 0 & B \\
0 & C & D \\
0 & 0 & E
\end{array}\right]\left[\begin{array}{ccc}
0 & \sqrt{2} X_{j} & I \\
0 & X_{j} & 0 \\
0 & 0 & I
\end{array}\right]=\left[\begin{array}{ccc}
0 & \sqrt{2} A X_{j} & A+B \\
0 & C X_{j} & D \\
0 & 0 & E
\end{array}\right]=\left[\begin{array}{ccc}
0 & Y_{j} & 0 \\
0 & 0 & I \\
0 & 0 & 0
\end{array}\right] .
$$

Thus, $D=I, E=0$, and $C X_{j}=0$. In the interest of minimizing the norm of $A_{j+1}$, we may as well take $C=0$ ( $C$ appears in a column whose other entries are 0's, so the norm cannot be made larger by this choice). If $A_{j}$ is an operator in $A \lg \mathcal{L}_{j}$ for which $A_{j} X_{j}=Y_{j}$, we can take $A=(1 / \sqrt{2}) A_{j}$. We may also assume that $A_{j}$ has, among all solutions of $Q X_{j}=Y_{j}$ in $A \lg \mathcal{L}_{j}$, the minimal norm. Thus, we can suppose that

$$
A_{j+1}=\left[\begin{array}{ccc}
(1 / \sqrt{2}) A_{j} & 0 & -(1 / \sqrt{2}) A_{j} \\
0 & 0 & I \\
0 & 0 & 0
\end{array}\right] .
$$

Furthermore, if $A_{j} \in A \lg \mathcal{L}_{j}$, then $A_{j+1} \in A \lg \mathcal{L}_{j+1}$. There may be other solutions in $A \lg \mathcal{L}_{j+1}$, but - as we have argued - none can have smaller norm than this one.

Furthermore, the lemmas show that $\left\|A_{j+1}\right\|^{2} \geq \frac{1}{2}+\left\|A_{j}\right\|^{2}$; and, additionally, if $X_{j}^{*} E X_{j} \geq Y_{j}^{*} E Y_{j}$ for all $E \in \mathcal{L}_{j}$, then $X_{j+1}^{*} E X_{j+1} \geq Y_{j+1}^{*} E Y_{j+1}$ for all $E \in \mathcal{L}_{j+1}$.

Thus, we have a sequence of commutative subspace lattices and operators $X_{j}$ and $Y_{j}$ such that, if $A_{j} \in A \lg \mathcal{L}_{j}$ and if $A_{j} X_{j}=Y_{j}$, then $\left\|A_{j}\right\|^{2} \geq(n+1) / 2$. (The latter fact follows because $\left\|A_{1}\right\| \geq 1$.) Note also that, for each $j,\left\|X_{j}\right\| \geq\left\|Y_{j}\right\|$. Denote as follows:

$$
\mathcal{L}=\sum_{1}^{\infty} \oplus \mathcal{L}_{j}, \quad X=\sum \oplus \frac{X_{j}}{\left\|X_{j}\right\|}, \quad Y=\sum \oplus \frac{Y_{j}}{\left\|X_{j}\right\|}
$$


Then $\mathcal{L}$ is a commutative subspace lattice and the operators $X$ and $Y$ are bounded. If $A \in A \lg \mathcal{L}$, then $A$ must break up as a direct sum in the same way as $X$ and $Y$; that is, $A=\sum \oplus A_{j}$, where $A_{j} \in A \lg \mathcal{L}_{j}$. If $A X=Y$, then, for each $j$, we need $A_{j} X_{j} /\left\|X_{j}\right\|=Y_{j} /\left\|X_{j}\right\|$, or $A_{j} X_{j}=Y_{j}$. But then $\left\|A_{j}\right\|^{2} \geq \frac{n+1}{2}$, and so $A$ cannot be a bounded operator. This finishes the argument.

An example such as this one leaves open a few questions. For instance: The lattice above has infinite width - that is, there is not a finite collection of mutually commuting nests which generate it. Is it possible that, for a lattice of width $n$, there is a constant $K$ so that, whenever $\rho(X, Y)<\infty$, then there is an operator $A \in A \lg \mathcal{L}$ such that $A X=Y$ and $\|A\| \leq K \cdot \rho(X, Y)$ ? The result in [4] shows that, if $n=1$, the answer is 'yes' and that $K$ can be chosen to be 1 . However, even if $n=2$, we don't have either a proof or a counterexample.

Here is another question: Since the quantity $\rho(X, Y)$ does not, by itself, give us enough information to determine the existence of an interpolating operator, is there some additional information that would suffice? In this case, we have a candidate, a conjecture, and a proof that the conjecture is "almost" correct. As before, let $\mathcal{H}$ be a Hilbert space, let $\mathcal{L}$ be a CSL, and let $X$ and $Y$ be operators on $\mathcal{H}$. Suppose that there is an operator $A \in A l g \mathcal{L}$ such that $A X=Y$. Let $\mathcal{H}^{(n)}$ represent the Hilbert space $\mathcal{H} \otimes \mathbb{C}^{n}$, which may be thought of as a direct sum of $n$ copies of the original space. For any operator $B \in B(\mathcal{H})$, let $B^{(n)}$ represent the inflated operator $B \otimes I_{n}$, where $I_{n}$ is the identity operator on $\mathbb{C}^{n}$. Let the notations $\mathcal{H}^{(\infty)}$ and $B^{(\infty)}$ be defined similarly, where the original space is tensored with a separable infinite-dimensional space. If the operator $A$ lies in $A \lg \mathcal{L}$, then $A^{(n)}$ lies in $\operatorname{Alg} \mathcal{L} \otimes I_{n}$. It follows from work of K.J. Harrison [2] that, if the lattice $\mathcal{L}$ is completely distributive, then $\operatorname{Alg} \mathcal{L} \otimes I_{n}=\operatorname{Alg}\left(\mathcal{L} \otimes \operatorname{Lat}\left(I_{n}\right)\right)=\operatorname{Alg}\left(\mathcal{L} \otimes \mathcal{P}_{n}\right)$, where $\mathcal{P}_{n}$ represents the collection of all projections acting on $n$-dimensional space; we allow the possibility that $n=\infty$, in which case we mean $\mathcal{P}_{\infty}$ to act on a separable infinite-dimensional space. Of course, $\mathcal{P}_{n}$ and $\operatorname{Lat}\left(\operatorname{Alg} \mathcal{L} \otimes I_{n}\right)$ are not commutative lattices, so none of the known results about CSL algebras will apply. Obviously, if $A X=Y$, then $A^{(n)} X^{(n)}=Y^{(n)}$, and, moreover, $\left\|A^{(n)}\right\|=\|A\|$. For each $n$, including $n=\infty$, let

$$
\rho_{n}(X, Y)=\sup \left\{\frac{\left\|E^{\perp} Y^{(n)} f\right\|}{\left\|E^{\perp} X^{(n)} f\right\|}: E \in \operatorname{Lat}\left(\operatorname{Alg} \mathcal{L} \otimes I_{n}\right) \text { and } f \in \mathcal{H}^{(n)}\right\} .
$$

Note that, if $A X=Y$, then $\rho_{n}(X, Y) \leq\left\|A^{(n)}\right\|=\|A\|$ for each $n$. Therefore, if the operator $A$ exists, the collection $\left\{\rho_{n}(X, Y)\right\}$ is uniformly bounded. It is also clear that, whether there is an interpolating operator or not, $\rho_{n}(X, Y) \leq \rho_{n+1}(X, Y)$ for all $n$. Thus, we have

$$
\rho_{1}(X, Y) \leq \rho_{2}(X, Y) \leq \cdots \leq \lim _{n \rightarrow \infty} \rho_{n}(X, Y) \leq \rho_{\infty}(X, Y) .
$$

The condition $\rho_{\infty}(X, Y)<\infty$ is necessary for the existence of the interpolating operator $A$, and this condition is, at least ostensibly, stronger than the first-level condition $\rho(X, Y)=\rho_{1}(X, Y)<\infty$. In fact, the word "ostensibly" in the last sentence can be removed; for the example given above, we have $\rho_{1}(X, Y)=1$, but $\rho_{\infty}(X, Y)=\infty$.

Conjecture. If $\rho_{\infty}(X, Y)<\infty$, then there is an operator $A$ in $\operatorname{Alg} \mathcal{L}$ such that $\|A\|=\rho_{\infty}(X, Y)$ and $A X=Y$. 
A weaker conjecture is that a solution of $A X=Y$ exists if $\lim _{n \rightarrow \infty} \rho_{n}(X, Y)<$ $\infty$. We don't know whether these two conjectures are equivalent. However, the first conjecture is "almost" correct, in the sense that, if $\rho_{\infty}(X, Y)<\infty$, then $Y$ can be approximated by operators of the form $A X$. To be precise, $Y$ lies in the weak-* closure of the set $\left\{A X: A \in A \lg \mathcal{L}\right.$ and $\left.\|A\| \leq \rho_{\infty}(X, Y)\right\}$. The following argument is similar to one by Arveson [1].

Suppose that $\rho_{\infty}(X, Y)<\infty$. We want to show that the operator $Y$ lies in the weak-* closure of the set named above. Let $[A \lg \mathcal{L}] X$ represent the weak-* closure of the set $\{A X: A \in A \lg \mathcal{L}\}$. This set is a weak-* closed subspace of $B(\mathcal{H})$. Suppose that $\phi$ is a weak-* continuous linear functional defined on $B(\mathcal{H})$ that annihilates $[A \lg \mathcal{L}] X$. Then $\phi(A X)=0$ for all $A \in A \lg \mathcal{L}$. Now, $\phi$ is given by a trace-class operator, say $C: \phi(B)=\operatorname{tr}(B C)$ for all $B \in B(\mathcal{H})$. $C$ can always be written as $C=\sum x_{i} \otimes y_{i}^{*}$, where the summation converges in the trace-class norm and the notation $x \otimes y^{*}$ means the rank-one operator given by $\left(x \otimes y^{*}\right) f=\langle f, y\rangle x$. (To see that any trace-class operator can be written this way, use the polar decomposition and diagonalize the positive factor.) Furthermore, we can assume that $\sum\left\|x_{i}\right\|^{2}$ and $\sum\left\|y_{i}\right\|^{2}$ are both finite. With this decomposition, we can write, for any $A \in A \lg \mathcal{L}$,

$$
\begin{aligned}
\operatorname{tr}(A X C) & =\operatorname{tr}\left(A X\left(\sum x_{i} \otimes y_{i}^{*}\right)\right) \\
& =\operatorname{tr}\left(\sum A X\left(x_{i} \otimes y_{i}^{*}\right)\right) \\
& =\operatorname{tr}\left(\sum\left((A X) x_{i} \otimes y_{i}^{*}\right)\right) \\
& =\sum \operatorname{tr}\left((A X) x_{i} \otimes y_{i}^{*}\right) \\
& =\sum\left\langle A X x_{i}, y_{i}\right\rangle \\
& =\left\langle A^{(\infty)} X^{(\infty)} \xi, \eta\right\rangle
\end{aligned}
$$

where $\xi$ and $\eta$ represent the vectors in $\mathcal{H}^{(\infty)}$ with components $\left\langle x_{1}, x_{2}, x_{3}, \ldots\right\rangle$ and $\left\langle y_{1}, y_{2}, y_{3}, \ldots\right\rangle$ respectively. Let $P$ be the orthogonal projection on $\mathcal{H}^{(\infty)}$ with range equal to the (norm) closure of the set $\left\{A^{(\infty)} X^{(\infty)} \xi: A \in A \lg \mathcal{L}\right\}$. The fact that $\operatorname{tr}(A X C)=0$ for all $A \in A \lg \mathcal{L}$ says that $\eta \in P^{\perp}$. Now, it is clear that $P$ is invariant for all $A^{(\infty)}$ with $A \in A \lg \mathcal{L}$, and, moreover, $X^{(\infty)} \xi \in P$. Therefore, $P$ is one of the projections which contributes to $\rho_{\infty}$ and we have $\left\|P^{\perp} Y^{(\infty)} \xi\right\| \leq\left\|P^{\perp} X^{(\infty)} \xi\right\|$. However, the last expression is zero, and hence $Y^{(\infty)} \xi \in P$. However, this is the same as saying that $\phi(Y)=0$. Therefore, no weak-* continuous linear functional can separate $Y$ from the set $[\operatorname{Alg} \mathcal{L}] X$ and we are done.

We conclude with a question. We have been trying to solve the equation $A X=$ $Y$, with $A$ in a CSL algebra. Hopenwasser's result shows that, in case $X$ has rank one, the condition $\rho(X, Y)<\infty$ is sufficient. On the other hand, if $X$ has dense range, then there is at most one operator $A$ such that $A X=Y$, and it is not hard to see that the condition $\rho(X, Y)<\infty$ ensures that $A$ lies in the required algebra. Therefore, in the extreme cases that the range of $X$ has dimension 1 or co-dimension 0 , the condition on $\rho(X, Y)$ is sufficient. Observe that in our main example above, the operator $X$ has infinite rank and co-rank. Is it possible that, if the rank or co-rank of $X$ is assumed to be finite, then the condition $\rho(X, Y)<\infty$ will ensure the existence of an interpolating operator $A$ (perhaps with norm larger than $\rho(X, Y))$ in $\operatorname{Alg} \mathcal{L}$ ? We will examine these questions in a subsequent paper. 


\section{REFERENCES}

1. W. Arveson, Interpolation Problems in Nest Algebras, Journal of Functional Analysis 20 (1975), 208-233. MR 52:3979

2. K. J. Harrison, The Tensor Product Formula for Reflexive Subspace Lattices, Canadian Mathematics Bulletin 38, number 3 (1995), 308-316. MR 96g: 47042

3. A. Hopenwasser, The Equation $T x=y$ in a Reflexive Operator Algebra, Indiana University Mathematics Journal 29 (1980). MR 81c:47014

4. E. Katsoulis, R. Moore, and T. Trent, Interpolation in Nest Algebras and Applications to Operator Corona Theorems, Journal of Operator Theory 29 (1993), 115-123. MR 95b:47052

5. E. C. Lance, Some Properties of Nest Algebras, Proceedings of the London Mathematical Society 3, number 19 (1969), 45-68. MR 39:3325

Department of Mathematics, University of Alabama, Tuscaloosa, Alabama 354870350

E-mail address: rmoore@gp.as.ua.edu 